Some properties of generalized fractional integral with Lengendre functions kernel's

M. M. Rodrigues

Citation: AIP Conference Proceedings 1637, 882 (2014); doi: 10.1063/1.4904660

View online: $\mathrm{http}: / / \mathrm{dx}$.doi.org/10.1063/1.4904660

View Table of Contents: http://aip.scitation.org/toc/apc/1637/1

Published by the American Institute of Physics 


\title{
Some properties of generalized fractional integral with Lengendre functions kernel's
}

\author{
M. M. Rodrigues \\ Center for Research and Development in Mathematics and Applications, Department of Mathematics, \\ University of Aveiro, 3810-193 Aveiro, Portugal
}

\begin{abstract}
In this paper we introduce two integral transforms involving the Legendre function in the kernel (see the operators $I_{0^{+}}^{\alpha, \beta, \mu, \nu}$ and $I_{-}^{\alpha, \beta, \mu, v}$ defined below) which generalize the classical Liouville fractional integrals. Then, we study their boundedness as operators mapping the space $\mathscr{L}_{v, r}$ into the spaces $\mathscr{L}_{v-\alpha, r}$. Moreover, we calculate the Mellin transform of the fractional integrals presented in this paper.
\end{abstract}

Keywords: Integral transforms, Fractional integrals, Operational calculus, Legendre functions, Mellin transform

PACS: $02.30 . \mathrm{Uu}, 02.30 . \mathrm{Rz}, 02.30 . \mathrm{Vv}$

\section{INTRODUCTION}

Introduction in the 17th century by Leibnitz, when he described the derivative of order $\frac{1}{2}$, the fractional calculus has become a new attractive theory to the mathematicians, as well for other researcher of non-mathematician areas (biology, economy, physics, chemistry, etc). There are many references regarding the theory and developments of the fractional calculus, for example, the Samko's book [14] which provides a comprehensive study of this topic. We remark that during the years a large number of fractional derivatives were introduced, for example, Riemann-Liouville, Grunwald-Letnikov, Riesz, Caputo, among others.

One area of mathematics where fractional calculus plays nowadays an important role, is the one devoted to the study of special functions and integrals transforms, for example, the Lengendre functions.

One of the varieties of special functions which are encountered in the solution of physical problems is the class of functions called Legendre polynomials. For applications of Legendre polynomials in fluid dynamics to study the flow around the outside of a puff of hot gas rising through the air, see e.g. [2].

The Legendre functions are denoted by $P_{v}, Q_{v}$ and by $P_{v}^{\mu}, Q_{v}^{\mu}$ the associated Legendre functions which are generalizations of Legendre polynomials to non-integer degree. Moreover, the associated Legendre functions are solutions of the general Legendre equation:

$$
\left(1-x^{2}\right) \frac{d^{2} y}{d x^{2}}-2 x \frac{d y}{d x}+\left(v(v+1)-\frac{\mu^{2}}{1-x^{2}}\right) y=0
$$

where the complex numbers $v$ and $\mu$ are called the degree and order of the associated Legendre functions, respectively.

The Legendre transform is a powerful tool in theoretical physics and plays an important role in classical mechanics, statistical mechanics, and thermodynamics (see e.g.[9], [5], [6], [7], [8]).

Churchill introduced in [3] the Legendre transform of a function and developed its operational calculus. In [4] the authors introduced the associated Legendre transform,

$$
T_{n}^{m}\{f(x)\}=\int_{-1}^{1}\left(1-x^{2}\right)^{-\frac{m}{2}} P_{n}^{m}(x) f(x) d x, n, m \in \mathbb{N},
$$

as a generalization of the Legendre transform of Churchill.

In [10] the authors study some properties of generalized associated Legendre functions of first and second kind $P_{k}^{m, n}(z)$ and $Q_{k}^{m, n}(z), k, n, m \in \mathbb{N}$. Applying these functions, they introduced an integral transform. 
In this work, we consider the integral transforms defined, for $x>0$, by

$$
\begin{aligned}
& \left(I_{0^{+}}^{\alpha, \beta, \mu, v} f\right)(x)=\frac{x^{1-\beta+\mu}}{\Gamma(\alpha)} \int_{0}^{x}(x-t)^{\alpha-1} t^{\beta-1}\left(x^{2}-t^{2}\right)^{-\frac{\mu}{2}} P_{v}^{\mu}(c(t, x)) f(t) d t \\
& \left(I_{-}^{\alpha, \beta, \mu, v} f\right)(x)=\frac{x^{1-\beta+\mu}}{\Gamma(\alpha)} \int_{x}^{\infty}(t-x)^{\alpha-1} t^{\beta-1}\left(t^{2}-x^{2}\right)^{-\frac{\mu}{2}} P_{v}^{\mu}(c(t, x)) f(t) d t .
\end{aligned}
$$

When $\mu=0, \beta=1$ and $c(t, x)=1$, the integrals (2) and (3) coincide with the classical Liouville fractional integrals [14]

$$
\begin{aligned}
\left(I_{0^{+}}^{\alpha, \beta, \mu, v} f\right)(x) \equiv\left(I_{0^{+}}^{\alpha} f\right)(x)=\frac{1}{\Gamma(\alpha)} \int_{0}^{x}(x-t)^{\alpha-1} f(t) d t, & x>0, \\
\left(I_{-}^{\alpha, \beta, \mu, v} f\right)(x) \equiv\left(I_{-}^{\alpha} f\right)(x)=\frac{1}{\Gamma(\alpha)} \int_{x}^{\infty}(t-x)^{\alpha-1} f(t) d t, & x>0 .
\end{aligned}
$$

Our paper is devoted to the study the generalized fractional integral transforms (2) and (3), and two of their modifications in the space $\mathscr{L}_{v, r}$ of the complex value Lebesgue measurable functions $f$ on $\mathbb{R}_{+}$such that for $v \in \mathbb{R}$

$$
\begin{aligned}
\|f\|_{v, r} & =\left(\int_{0}^{\infty}\left|t^{v} f(t)\right|^{r} \frac{d t}{t}\right)^{1 / r}<\infty, \quad 1 \leq r<\infty, \\
\|f\|_{v, \infty} & =\operatorname{ess} \sup _{t>0}\left(t^{v}|f(t)|\right)<\infty .
\end{aligned}
$$

\section{EXPLICIT BOUNDEDNESS FOR FRACTIONAL INTEGRAL TRANSFORMS}

In this section, we obtain the boundedness of the fractional integral transforms (2-3) as operators mapping the space $\mathscr{L}_{v, r}$ into the spaces $\mathscr{L}_{v-\alpha, r}$.

Theorem 0.1 Let $\alpha>0, \beta>v, \mu>2 \beta-2 v, c(t, x)=\frac{t}{x}$, and $1 \leq r \leq \infty$.

The operator $I_{0^{+}}^{\alpha, \beta, \mu, v}$ is bounded from $\mathscr{L}_{v, r}$ into $\mathscr{L}_{v-\alpha, r}$ and

$$
\left\|I_{0^{+}}^{\alpha, \beta, \mu, v} f\right\|_{v-\alpha, r} \leq C_{\alpha, \beta, \mu, v, v}\|f\|_{v, r}
$$

where

$$
C_{\alpha, \beta, \mu, v, v}=\frac{1}{\Gamma(\alpha)} \Gamma\left[\begin{array}{cc}
\beta-v & \alpha-\mu \\
1-\mu & \alpha+\beta-v-\mu
\end{array}\right] 3^{F} 2\left(-v, 1+v, \alpha-\mu ; 1-\mu, \alpha+\beta-v-\mu ; \frac{1}{2}\right) .
$$


Proof: Let $1 \leq r<\infty$. Using (6) and making the change of variable $t=u x$, we obtain

$$
\begin{aligned}
\left\|I_{0^{+}}^{\alpha, \beta, \mu, v} f\right\|_{v-\alpha, r} & =\left(\int_{0}^{\infty}\left|x^{v-\alpha}\left(I_{0^{+}}^{\alpha, \beta, \mu, v} f\right)(x)\right|^{r} \frac{d x}{x}\right)^{1 / r} \\
& =\left(\int_{0}^{\infty}\left|x^{v-1} \frac{x^{-\alpha-\beta+\mu+2}}{\Gamma(\alpha)} \int_{0}^{x}(x-t)^{\alpha-1} t^{\beta-1}\left(x^{2}-t^{2}\right)^{-\frac{\mu}{2}} P_{v}^{\mu}\left(\frac{t}{x}\right) f(t) d t\right|^{r} \frac{d x}{x}\right)^{1 / r} \\
& =\left(\int_{0}^{\infty}\left|x^{v-\frac{1}{r}} \frac{1}{\Gamma(\alpha)} \int_{0}^{1}(1-u)^{\alpha-1} u^{\beta-1}\left(1-u^{2}\right)^{-\frac{\mu}{2}} P_{v}^{\mu}(u) f(u x) d u\right|^{r} d x\right)^{1 / r} \\
& \leq \int_{0}^{1}\left(\int_{0}^{\infty}\left|\frac{x^{\nu-\frac{1}{r}}}{\Gamma(\alpha)}(1-u)^{\alpha-1} u^{\beta-1}\left(1-u^{2}\right)^{-\frac{\mu}{2}} P_{v}^{\mu}(u) f(u x)\right| d x\right)^{1 / r} d u \\
& \leq \int_{0}^{1} \frac{1}{\Gamma(\alpha)}(1-u)^{\alpha-1} u^{\beta-v-1}\left(1-u^{2}\right)^{-\frac{\mu}{2}}\left|P_{v}^{\mu}(u)\right|\left(\int_{0}^{\infty}\left|t^{\nu} f(t)\right|^{r} \frac{d t}{t}\right)^{1 / r} d u \\
& =\|f\|_{v, r} \frac{1}{\Gamma(\alpha)} \int_{0}^{1}(1-u)^{\alpha-1} u^{\beta-v-1}\left(1-u^{2}\right)^{-\frac{\mu}{2}}\left|P_{v}^{\mu}(u)\right| d u . .
\end{aligned}
$$

From relation (2.17.5.6) in [12], we have

$$
\begin{aligned}
C_{\alpha, \beta, \mu, v, v} & =\frac{1}{\Gamma(\alpha)} \int_{0}^{1}(1-u)^{\alpha-1} u^{\beta-v-1}\left(1-u^{2}\right)^{-\frac{\mu}{2}} P_{v}^{\mu}(u) d u \\
& =\frac{1}{\Gamma(\alpha)} \Gamma\left[\begin{array}{cc}
\beta-v & \alpha-\mu \\
1-\mu & \alpha+\beta-v-\mu
\end{array}\right] 3^{F} 2\left(-v, 1+v, \alpha-\mu ; 1-\mu, \alpha+\beta-v-\mu ; \frac{1}{2}\right) .
\end{aligned}
$$

For $r=\infty$ we have

$$
\begin{aligned}
\left|x^{v-\alpha} I_{0^{+}}^{\alpha, \beta, \mu, v}\right| & =\left|x^{v-\alpha} \frac{x^{1-\beta+\mu}}{\Gamma(\alpha)} \int_{0}^{x}(x-t)^{\alpha-1} t^{\beta-1}\left(x^{2}-t^{2}\right)^{-\frac{\mu}{2}} P_{v}^{\mu}\left(\frac{t}{x}\right) f(t) d t\right| \\
& =\left|x^{v-1} \frac{x^{-\alpha-\beta+\mu+2}}{\Gamma(\alpha)} \int_{0}^{x}(x-t)^{\alpha-1} t^{\beta-1}\left(x^{2}-t^{2}\right)^{-\frac{\mu}{2}} P_{v}^{\mu}\left(\frac{t}{x}\right) f(t) d t\right| \\
& \leq \frac{x^{v-1}}{\Gamma(\alpha)} \int_{0}^{1}(1-u)^{\alpha-1} u^{\beta-1}\left(1-u^{2}\right)^{-\frac{\mu}{2}}\left|P_{v}^{\mu}(u)\right|\left|t^{-v}\left(t^{v} f(t)\right)\right| x d u \\
& \leq\|f\|_{v, \infty} \frac{1}{\Gamma(\alpha)} \int_{0}^{1}(1-u)^{\alpha-1} u^{\beta-v-1}\left(1-u^{2}\right)^{-\frac{\mu}{2}}\left|P_{v}^{\mu}(u)\right| d u \\
& =\|f\|_{v, \infty} C_{\alpha, \beta, \mu, v, v} .
\end{aligned}
$$

This completes the proof.

Theorem 0.2 Let $\alpha>\mu, \alpha+\beta<\max \{v+\mu+v+2, v+\mu-v+1\}, c(t, x)=\frac{t}{x}$, and $1 \leq r \leq \infty$. The operator $I_{-}^{\alpha, \beta, \mu, v}$ is bounded from $\mathscr{L}_{v, r}$ into $\mathscr{L}_{v-\alpha, r}$ and

$$
\left\|I_{-}^{\alpha, \beta, \mu, v} f\right\|_{v-\alpha, r} \leq C_{\alpha, \beta, \mu, v, v}^{*}\|f\|_{v, r} .
$$


Proof: Let $1 \leq r<\infty$. Using (6) and making the change of variable $t=x u$ and similarly to what was done in the previous theorem

$$
\begin{aligned}
\left\|I_{-}^{\alpha, \beta, \mu, v} f\right\|_{v-\alpha, r} & =\left(\int_{0}^{\infty}\left|x^{\nu-\alpha}\left(I_{-}^{\alpha, \beta, \mu, v} f\right)(x)\right|^{r} \frac{d x}{x}\right)^{1 / r} \\
& =\left(\int_{0}^{\infty}\left|x^{\nu-1} \frac{x^{-\alpha-\beta+\mu+2}}{\Gamma(\alpha)} \int_{x}^{\infty}(t-x)^{\alpha-1} t^{\beta-1}\left(t^{2}-x^{2}\right)^{-\frac{\mu}{2}} P_{v}^{\mu}\left(\frac{t}{x}\right) f(t) d t\right|^{r} \frac{d x}{x}\right)^{1 / r} \\
& =\left(\int_{0}^{\infty}\left|x^{\nu-\frac{1}{r}} \frac{1}{\Gamma(\alpha)} \int_{1}^{\infty}(u-1)^{\alpha-1} u^{\beta-1}\left(u^{2}-1\right)^{-\frac{\mu}{2}} P_{v}^{\mu}(u) f(u x) d u\right|^{r} d x\right)^{1 / r} \\
& \leq \int_{1}^{\infty}\left(\int_{0}^{\infty}\left|\frac{x^{v-\frac{1}{r}}}{\Gamma(\alpha)}(u-1)^{\alpha-1} u^{\beta-1}\left(u^{2}-1\right)^{-\frac{\mu}{2}} P_{v}^{\mu}(u) f(u x)\right|^{r} d x\right)^{1 / r} d u \\
& \leq \int_{1}^{\infty} \frac{1}{\Gamma(\alpha)}(u-1)^{\alpha-1} u^{\beta-v-1}\left(u^{2}-1\right)^{-\frac{\mu}{2}}\left|P_{v}^{\mu}(u)\right|\left(\int_{0}^{\infty}\left|t^{v} f(t)\right|^{r} \frac{d t}{t}\right)^{1 / r} d u \\
& =\|f\|_{v, r} \frac{1}{\Gamma(\alpha)} \int_{1}^{\infty}(u-1)^{\alpha-1} u^{\beta-v-1}\left(u^{2}-1\right)^{-\frac{\mu}{2}}\left|P_{v}^{\mu}(u)\right| d u .
\end{aligned}
$$

From relation (2.17.5.11) in [12], we have

$$
\begin{aligned}
C_{\alpha, \beta, \mu, v, v}^{*} & =\frac{1}{\Gamma(\alpha)} \int_{1}^{\infty}(u-1)^{\alpha-1} u^{\beta-v-1}\left(u^{2}-1\right)^{-\frac{\mu}{2}} P_{v}^{\mu}(u) d u \\
& =\frac{1}{\Gamma(\alpha)}\left\{\Gamma\left[\begin{array}{cc}
\alpha-\mu & v+1-\beta-\alpha+\mu \\
v+1-\beta & 1-\mu
\end{array}\right] 3^{F} 2\left(\alpha-\mu,-v, 1+v ; \alpha+\beta-v-\mu, 1-\mu ; \frac{1}{2}\right)\right. \\
& -\frac{\sin (v \pi)}{\pi} 2^{\alpha+\beta-\mu-v-1} \Gamma\left[\begin{array}{cc}
\alpha+\beta-\mu-v-1 & v-\alpha-\beta+\mu-v+1 \\
v-\alpha-\beta-2 & v-\beta-\alpha+\mu-v+2
\end{array}\right] \\
& \left.\times 3^{F} 2\left(v+1-\beta, v+\mu-\alpha-\beta-v+1, v+\mu-\alpha-\beta+v+2 ; v-\alpha-\beta+2, v+\mu-\alpha-\beta+2 ; \frac{1}{2}\right)\right\} .
\end{aligned}
$$

For $r=\infty$ we have

$$
\begin{aligned}
\left|x^{v-\alpha} I_{-}^{\alpha, \beta, \mu, v}\right| & =\left|x^{\nu-\alpha} \frac{x^{1-\beta+\mu}}{\Gamma(\alpha)} \int_{x}^{\infty}(t-x)^{\alpha-1} t^{\beta-1}\left(t^{2}-x^{2}\right)^{-\frac{\mu}{2}} P_{v}^{\mu}\left(\frac{t}{x}\right) f(t) d t\right| \\
& \leq \frac{x^{v-1}}{\Gamma(\alpha)} \int_{x}^{\infty}(u-1)^{\alpha-1} u^{\beta-1}\left(u^{2}-1\right)^{-\frac{\mu}{2}}\left|P_{v}^{\mu}(u)\right| x\left|t^{-v}\left(t^{v} f(t)\right)\right| d u \\
& \leq\|f\|_{v, \infty} \frac{1}{\Gamma(\alpha)} \int_{x}^{\infty}(u-1)^{\alpha-1} u^{\beta-v-1}\left(u^{2}-1\right)^{-\frac{\mu}{2}}\left|P_{v}^{\mu}(u)\right| d u \\
& =\|f\|_{v, \infty} C_{\alpha, \beta, \mu, v, v}^{*} .
\end{aligned}
$$

This completes the proof.

\section{GENERALIZED EXPLICIT BOUNDEDNESS FOR FRACTIONAL INTEGRAL TRANSFORMS}

Now, we want to obtain generalized boundedness of the fractional integral transforms (2-3) as operators mapping the space $\mathscr{L}_{v, r}$ into the spaces $\mathscr{L}_{v-\alpha, r}$. To so, we introduce a technical Lemma. 
Lemma 0.3 Let $G, \tilde{G}, h: \mathbb{R} \rightarrow \mathbb{C}$ be measurable. Let $s \in \mathbb{R} \backslash\{0\}$. Let

$$
H_{+}(x)=\int_{0}^{x} G(t / x) h(t) d t \quad \text { and } \quad H_{-}(x)=\int_{x}^{\infty} \tilde{G}(t / x) h(t) d t \quad \forall x>0 .
$$

Let $w \in \mathbb{R}$. Let $1 \leq r \leq+\infty$. Then

$$
\left\|H_{+}\right\|_{w-1, r} \leq\left(\int_{0}^{1} u^{-w}|G(u)| d u\right)\|h\|_{w, r} \quad \text { and } \quad\left\|H_{-}\right\|_{w-1, r} \leq\left(\int_{1}^{\infty} u^{w}|\tilde{G}(u)| d u\right)\|h\|_{w, r} .
$$

\section{Proof:}

Without loss of generality one can assume that the functions $G, \tilde{G}$ and $h$ are real and non-negative.If $r<+\infty$, then, by taking $t=u x$ in the inner integral, by applying the generalized Hölder inequality, and by finally taking $u=t / x$ in the inner integral one has

$$
\begin{gathered}
{\left[\int_{0}^{\infty}\left(x^{w-1} H_{+}(x)\right)^{r} \frac{d x}{x}\right]^{1 / r}=\left[\int_{0}^{\infty}\left(\int_{0}^{1} x^{w} G(u) h(u x) d u\right)^{r} \frac{d x}{x}\right]^{1 / r}} \\
\leq \int_{0}^{1}\left[\int_{0}^{\infty}\left(x^{w} G(u) h(u x)\right)^{r} \frac{d x}{x}\right]^{1 / r} d u=\int_{0}^{1} G(u) u^{-w} d u\left[\int_{0}^{\infty}\left(t^{w} h(t)\right)^{r} \frac{d t}{t}\right]^{1 / r} .
\end{gathered}
$$

Thus, the first inequality in (10) is proved for $r<+\infty$. The proof of the second inequality for $r<+\infty$ is similar and is accordingly omitted. If instead $r=+\infty$, then by applying the generalized Hölder inequality, and by finally taking $u=t / x$ in the inner integral, we obtain

$$
x^{w-1} \int_{0}^{x} G(t / x) h(t) d t=\int_{0}^{1} u^{-w} G(u)(u x)^{w} h(u x) d u \leq\left(\int_{0}^{1} u^{-w}|G(u)| d u\right)\|h\|_{w,+\infty}
$$

for all $x \in] 0,+\infty[$. Thus one deduces the validity of the first inequality in (10). The proof of the second inequality is similar and is accordingly omitted.

Remark: Now, we can see Theorem 0.1 as a particular case of Lemma 0.3. It follows from Lemma 0.3 with

$$
G(t / x) \equiv \frac{1}{|\Gamma(\alpha)|}\left(\frac{t}{x}\right)^{\beta-\alpha}\left(1-\frac{t}{x}\right)^{\alpha-1}\left(1-\left(\frac{t}{x}\right)^{2}\right)^{-\frac{\mu}{2}} P_{v}^{\mu}\left(\frac{t}{x}\right), \quad h(t) \equiv t^{\alpha-1} f(t),
$$

and $w=v-\alpha+1$. Similarly, we can see the Theorem 0.2 as a particular case of Lemma 0.3.

\section{MELLIN TRANSFORMS OF GENERALIZED FRACTIONAL OPERATORS}

Hjalmar Mellin(1854-1933), [13] for a summary of his works) gave his name to the Mellin transform, a close relative of the integral transforms of Laplace and Fourier.

The Mellin transform of a function $f(x)$ defined on the interval $[0,+\infty[$ is given by

$$
\mathscr{M}\{f(x)\}(s) \equiv \int_{0}^{\infty} x^{s-1} f(x) d x .
$$

In the following result we obtain the Mellin transforms of the generalized fractional integrals (2-3).

Theorem 0.4 Let $\alpha>\max \{\mu, 0\}$ and $c(t, x)=\frac{x}{t}$. Then,

$$
\begin{aligned}
& \mathscr{M}\left\{\left(I_{0^{+}}^{\alpha, \beta, \mu, v} f\right)(x)\right\}(s)=B_{\alpha, \beta, \mu, v}(s) \mathscr{M}\{f(t)\}(s+\alpha), s<\frac{1}{2}+\beta-\alpha \\
& \mathscr{M}\left\{\left(I_{-}^{\alpha, \beta, \mu, v} f\right)(x)\right\}(s)=B_{\alpha, \beta, \mu, v}^{*}(s) \mathscr{M}\{f(t)\}(s+\alpha), s>\beta-\mu-1
\end{aligned}
$$


where

$$
\begin{aligned}
& B_{\alpha, \beta, \mu, v}(s)=\frac{1}{\Gamma(\alpha)}\left\{\Gamma\left[\begin{array}{cc}
\alpha-\mu & \beta-\alpha-s \\
\beta-s-\mu & 1-\mu
\end{array}\right] 3^{F} 2\left(\alpha-\mu,-v, 1+v ; \alpha-\beta+s, 1-\mu ; \frac{1}{2}\right)\right. \\
& -\frac{\sin (v \pi)}{\pi} 2^{\alpha-\beta+s} \Gamma\left[\begin{array}{ccc}
\alpha-\beta+s & -\alpha+\beta-s-v & \beta-\alpha-s+v+1 \\
-\alpha+\beta-s-\mu+1 &
\end{array}\right] \\
& \left.\times \quad 3^{F} 2\left(\beta-s-\mu,-\alpha+\beta-s-v, 1-\alpha+\beta-s+v ;-\mu-\alpha+\beta-s+1,-\alpha+\beta-s+1 ; \frac{1}{2}\right)\right\},
\end{aligned}
$$

and

$$
\begin{aligned}
B_{\alpha, \beta, \mu, v}^{*}(s) & =\frac{1}{\Gamma(\alpha)} \int_{0}^{1}(1-u)^{\alpha-1} u^{s-\beta+\mu}\left(1-u^{2}\right)^{-\frac{\mu}{2}} P_{v}^{\mu}(u) d u \\
& =\frac{1}{\Gamma(\alpha)} \Gamma\left[\begin{array}{cc}
s-\beta-\mu-1 & \alpha-\mu \\
1-\mu & \alpha-\beta+s+1
\end{array}\right] 3^{F} 2\left(-v, 1+v, \alpha-\mu ; 1-\mu, \alpha-\beta+s+1 ; \frac{1}{2}\right) .
\end{aligned}
$$

Proof: By the definition of Mellin transform (13) and (2), and using Fubini's theorem we have

$$
\begin{aligned}
\mathscr{M}\left\{\left(I_{0^{+}}^{\alpha, \beta, \mu, v} f\right)(x)\right\}(s) & =\int_{0}^{\infty} x^{s-1}\left(\frac{x^{1-\beta+\mu}}{\Gamma(\alpha)} \int_{0}^{x}(x-t)^{\alpha-1} t^{\beta-1}\left(x^{2}-t^{2}\right)^{-\frac{\mu}{2}} P_{v}^{\mu}\left(\frac{x}{t}\right) f(t) d t\right) d x \\
& =\frac{1}{\Gamma(\alpha)} \int_{0}^{\infty} t^{\beta-1} f(t) \int_{t}^{\infty}(x-t)^{\alpha-1} x^{s-\beta+\mu}\left(x^{2}-t^{2}\right)^{-\frac{\mu}{2}} P_{v}^{\mu}\left(\frac{x}{t}\right) d x d t \\
& =\frac{1}{\Gamma(\alpha)} \int_{0}^{\infty} t^{s-1+\alpha} f(t) \int_{1}^{\infty} u^{s-\beta+\mu}(u-1)^{\alpha-1}\left(u^{2}-1\right)^{-\frac{\mu}{2}} P_{v}^{\mu}(u) d u d t \\
& =\frac{1}{\Gamma(\alpha)} B_{\alpha, \beta, \mu, v}(s) \int_{0}^{\infty} t^{s-1+\alpha} f(t) d t \\
& =\frac{1}{\Gamma(\alpha)} B_{\alpha, \beta, \mu, v}(s) \mathscr{M}\{f(t)\}(s+\alpha)
\end{aligned}
$$

after using the change of variable $u=\frac{x}{t}$, and taking into account the relation (2.17.5.11) in [12] we obtain

$$
\begin{aligned}
& B_{\alpha, \beta, \mu, v}(s)=\frac{1}{\Gamma(\alpha)} \int_{1}^{\infty} u^{s-\beta+\mu}(u-1)^{\alpha-1}\left(u^{2}-1\right)^{-\frac{\mu}{2}} P_{v}^{\mu}(u) d u \\
& =\frac{1}{\Gamma(\alpha)}\left\{\Gamma\left[\begin{array}{cc}
\alpha-\mu & \beta-\alpha-s \\
\beta-s-\mu & 1-\mu
\end{array}\right] 3^{F} 2\left(\alpha-\mu,-v, 1+v ; \alpha-\beta+s, 1-\mu ; \frac{1}{2}\right)\right. \\
& -\frac{\sin (v \pi)}{\pi} 2^{\alpha-\beta+s} \Gamma\left[\begin{array}{ccc}
\alpha-\beta+s & -\alpha+\beta-s-v & \beta-\alpha-s+v+1 \\
-\alpha+\beta-s-\mu+1 &
\end{array}\right] \\
& \left.\times \quad 3^{F} 2\left(\beta-s-\mu,-\alpha+\beta-s-v, 1-\alpha+\beta-s+v ;-\mu-\alpha+\beta-s+1,-\alpha+\beta-s+1 ; \frac{1}{2}\right)\right\},
\end{aligned}
$$

for $s<\frac{1}{2}+\beta-\alpha$ and $\alpha-\beta+s-1<v<\beta-\alpha-s$. This proves (14).

The proof of (15) is similar.

\section{ACKNOWLEDGMENTS}

This work was supported by Portuguese funds through the CIDMA - Center for Research and Development in Mathematics and Applications, and the Portuguese Foundation for Science and Technology ("FCT-Fundação para a Ciência e a Tecnologia"), within project PEst-OE/MAT/UI4106/2014. 


\section{REFERENCES}

1. A. Erdélyi, W. Magnus, F. Oberhettinger and F.G. Tricomi, Tables of Integral Transforms-Vol II, Bateman Manuscript Project, California Institute of Technology, McGraw Hill, New York, 1954.

2. A. R. Paterson, A First Course in Fluid Dynamics, Cambridge University Press, Cambridge; New York, 1983.

3. R. V. Churchill, The operational Calculus of Legendre Transforms, J. Math., Phys. 33, 165-77 (1954).

4. L. Debrath and C.W. Harrell, The Operational Calculus of Associated Legendre Transform-1, Ind. J. Pure and App. Math. 7, 278-291 (1976).

5. C.C. Cheng, Maxwell's equations in dynamics, Am. J. Phys. 34, 622 (1966).

6. A. L. Fetter and J. D. Walecka, Theoretical Mechanics of Particles and Continua, McGraw-Hill, New York, 1980.

7. K. Huang, Statistical Mechanics, Wiley, New York, 1987.

8. H. S. Robertson, Statistical Thermophysics, Prentice Hall, New York, 1997.

9. J. W. Cannon, Connecting thermodynamics to students calculus, Am. J. Phys. 72, 753-Ú757 (2004).

10. I. A. Fedotova, On an integral transform with generalized associated Legendre functions, Journal of Mathematical Sciences 68(6), 759-765 (1994).

11. A.A. Kilbas and O,V. Skoromnik, Integral transforms with the Legendre function of the first kind in the kernels on spaces, Integral Transforms and Special Functions 20(9), 653-672 (2009).

12. A.P. Prudnikov, Yu.A. Brychkov and O.I. Marichev, Integrals and Series, Volume 3: More special functions, Gordon and Breach Publisher, New York, 1990.

13. E. Lindelöf, Robert Hjalmar Mellin, Advances in Mathematics, 61 (1993) i-vi (H. Mellin's notice and bibliography).

14. S. G. Samko, A. A. Kilbas, and O. I. Marichev, Fractional integrals and derivatives: theory and applications, Gordon and Breach, New York, 1993. 